\title{
Advances in nanotechnology, nanopollution, nanotoxicology, and nanomedicine
}

\author{
Lotfi Aleya $^{1} \cdot$ Mohamed M. Abdel-Daim ${ }^{2,3}$ \\ Published online: 20 April 2020 \\ (C) Springer-Verlag GmbH Germany, part of Springer Nature 2020
}

Nanotechnology is a science that deals with the design, characterization, synthesis, and application of nanoscale materials $(1-100 \mathrm{~nm})$. Nanomaterials (NMs) have unique physicochemical characteristics, including ultra-small size, large surface area to mass ratio, and high reactivity. Nanomedicine is the application of nanotechnology in medicine to advance diagnostic, preventive, and therapeutic strategies. The potent antioxidant properties of some NMs open the door to develop new therapies with enhanced, targeted actions. However, oxidative stress is the main adverse effect of metallic oxide NMs, such as nickel oxide, zinc oxide, and titanium dioxide. Therefore, NMs have double-edged effects on cellular redox hemostasis. Recently, many researchers have focused on the ecological and safety issues of synthetic NMs to reduce the risk of their toxicity. The present special issue "Advances in Nanotechnology, Nanopollution, Nanotoxicology and Nanomedicine (ANNNN)" is dedicated to integrate the previous and the current biological, medical, and ecological data on NMs.

- Elkeiy et al. isolated Chitosan NPs from Artemia salina and evaluated their anticancer activities in vitro (using

Responsible editor: Philippe Garrigues

Lotfi Aleya

lotfi.aleya@univ-fcomte.fr

1 Chrono-Environnement Laboratory, UMR CNRS 6249, Bourgogne Franche-Comté University, 25030 Besançon Cedex, France

2 Department of Zoology, Science College, King Saud University, Riyadh 11451, Saudi Arabia

3 Department of Pharmacology, Faculty of Veterinary Medicine, Suez Canal University, Ismailia 41522, Egypt
HepG2) and in vivo (diethylnitrosamine-induced hepatocellular carcinoma in rats) (Elkeiy et al. 2018).

- El-Sayed and Kamel and Zahin et al. reviewed the shape and structure of available NPs and their recent applications in medicine to revolutionize medical diagnostics, therapeutics, and prevention in both human and veterinary medicine (El-Sayed and Kamel 2018, 2019; Zahin et al. 2019).

- Elsharkawy et al. evaluated the resistance induced by Phoma sp. GS8-3 fungus or nanosilica against cucumber mosaic virus (CMV). They reported that nanosilica is as effective as GS8-3 fungus in upregulating CMV defense-related genes, especially heat shock proteins (Elsharkawy et al. 2018b).

- Another research by Elsharkawy et al. assessed the capability of zinc oxide NPs to protect tomatoes against Pseudomonas syringae bacteria. They concluded that zinc oxide NPs enhanced tomato plant growth, exhibited direct anti-bacterial effects, and increased the expression of genes involved in antimicrobial defense (Elsharkawy et al. 2018a).

- Similarly, another study by Hafez et al. has shown that dietary supplementation with zinc oxide NPs improved the immune response (as evidenced by increased serum IgY concentrations, total lymphocytes, and macrophages count) and antioxidant enzymatic activity in broiler chicken (Hafez et al. 2019).

- Barkia et al. examined the acute oral toxicity study of microalgal protein hydrolysates (MPH) from Bellerochea malleus on Wistar rats and concluded the overall safety of MPH providing a new evidence for their potential use in nutraceutical applications and functional foods (Barkia et al. 2019).

- Maher et al. reviewed the potential applications of synthetic melanin NPs based on their free radical scavenging activities and suggested that modern 
research techniques can further characterize these NPs to explore their full potential (Maher et al. 2019).

- A different review article by Sayour et al. enumerated the multiple advantages of surface-modified yttrium oxide NPs, including enhanced colloidal stability, free radical scavenging activity, and low risk of cytotoxicity. These advantages could pave the way towards their use as theranostics in neurodegenerative diseases (Sayour et al. 2019).

- Sharma et al. went further to explore the potential benefits of nanoinformatics and biological nanomodeling in designing novel anti-cancer therapies. These technologies can produce nanomedicines with enhanced and targeted effects (Sharma et al. 2019).

- El-Rouby et al. explored another beneficial effect of NPs. They showed that layered double hydroxide beads nanocomposites can act as efficient adsorbents for heavy metals as $\mathrm{Cd}^{2+}$ and $\mathrm{Cu}^{2+}$ ions from waste water. Furthermore, they assessed the effects of different factors as contact time, adsorbent weight, $\mathrm{pH}$ value, and heavy metal ion concentration on the adsorption efficacy (El Rouby et al. 2018).

- Abdeen and Ghoneim aimed to reduce the cost and improve the efficiency of $\mathrm{Mg}-\mathrm{Co}$ nanoferrites in crude oil adsorption in oily waste water treatment through blending chitosan hydrogel (Abdeen and Ghoneim 2018).

- In terms of nanotoxicity, Fadda et al. aimed to evaluate whether carnosine and melatonin can ameliorate titanium oxide NP-induced oxidative stress, inflammation, DNA damage, and apoptosis in rat liver. They showed promising prophylactic effects for both compounds in hepatoprotection against titanium oxide NP-induced hepatotoxicity (Fadda et al. 2019).

- In the same vein, Kandeil et al. evaluated whether the extract of Moringa seeds can alleviate the oxidative stress induced by titanium oxide NPs in rat brain and improve mitochondrial viability. They showed that Moringa seeds extract can exert these effects through stimulating Nrf2/NQO1 signaling and reducing tissue expression of pro-inflammatory markers, Fas, and caspase-3 (Kandeil et al. 2019).

- Another experiment by Sayed et al. investigated 4nonylphenol (4-NP)-induced genotoxicity in medaka (Oryzias latipes) erythrocytes. They further revealed that medaka were more sensitive to the genotoxic effects of 4 NP than catfish (Sayed et al. 2018).

- Al-Naggar et al. examined the sublethal effects of chronic exposure to $\mathrm{CdO}$ and/or $\mathrm{PbO}$ nanoparticles on the honey bee (Apis millefera L.) workers. They reported that both $\mathrm{CdO}$ and $\mathrm{PbO}$ NPs reduced the survival, feeding rate, acetylcholinesterase activity, and expression of stress-related detoxifying enzymes (Naggar et al. 2018).

- In a review article by Samak et al., the authors summarized the recent literature on the developmental toxicity of carbon, diamond, and graphene NPs during the early stages of chicken embryogenesis (Samak et al. 2018).

\section{References}

Abdeen ZI, Ghoneim AI (2018) Improving of the Mg-Co nanoferrites efficiency for crude oil adsorption from aqueous solution by blending them with chitosan hydrogel. Environ Sci Pollut Res:1-11

Barkia I, Bouaziz HK, Boudawara TS, Aleya L, Gargouri AF, Saari N (2019) Acute oral toxicity study on Wistar rats fed microalgal protein hydrolysates from Bellerochea malleus. Environmental Science and Pollution Research, 1-8

El-Sayed A, Kamel M (2018) Advanced applications of nanotechnology in veterinary medicine. Environmental Science and Pollution Research, 1-14

El-Sayed A, Kamel M (2019) Advances in nanomedical applications: diagnostic, therapeutic, immunization, and vaccine production. Environ Sci Pollut Res:1-14

El Rouby WM, El-Dek S, Goher ME, Noaemy SG (2018) Efficient water decontamination using layered double hydroxide beads nanocomposites. Environmental Science and Pollution Research, 1-19

Elkeiy MM, Khamis AA, El-Gamal MM, Gazia MMA, Zalat ZA, ElMagd MA (2018) Chitosan nanoparticles from Artemia salina inhibit progression of hepatocellular carcinoma in vitro and in vivo. Environ Sci Pollut Res:1-13

Elsharkawy M, Derbalah A, Hamza A, El-Shaer A (2018a) Zinc oxide nanostructures as a control strategy of bacterial speck of tomato caused by Pseudomonas syringae in Egypt. Environ Sci Pollut Res, 1-9

Elsharkawy MM, Suga H, Shimizu M (2018b) Systemic resistance induced by Phoma sp. GS8-3 and nanosilica against cucumber mosaic virus. Environ Sci Pollut Res, 1-9

Fadda LM, Ali HM, Mohamed AM, Hagar H (2019) Prophylactic administration of carnosine and melatonin abates the incidence of apoptosis, inflammation, and DNA damage induced by titanium dioxide nanoparticles in rat livers. Environ Sci Pollut Res, 1-9

Hafez A, Nassef E, Fahmy M, Elsabagh M, Bakr A, Hegazi E (2019) Impact of dietary nano-zinc oxide on immune response and antioxidant defense of broiler chickens. Environ Sci Pollut Res:1-7

Kandeil MA, Mohammed ET, Hashem KS, Aleya L, Abdel-Daim MM (2019): Moringa seed extract alleviates titanium oxide nanoparticles (TiO 2-NPs)-induced cerebral oxidative damage, and increases cerebral mitochondrial viability. Environ Sci Pollut Res, 1-16

Maher S, Mahmoud M, Rizk M, Kalil H (2019) Synthetic melanin nanoparticles as peroxynitrite scavengers, photothermal anticancer and heavy metals removal platforms. Environ Sci Pollut Res:1-12

Naggar YA, Dabour K, Masry S, Sadek A, Naiem E, Giesy JP (2018) Sublethal effects of chronic exposure to $\mathrm{CdO}$ or $\mathrm{PbO}$ nanoparticles or their binary mixture on the honey bee (Apis millefera L.). Environ Sci Pollut Res, 1-12

Samak DH, El-Sayed YS, Shaheen HM, Ali H, El-Hack MEA, Noreldin AE, El-Naggar K, Abdelnour SA, Saied EM, El-Seedi HR (2018) Developmental toxicity of carbon nanoparticles during embryogenesis in chicken. Environ Sci Pollut Res:1-15

Sayed AE-DH, Watanabe-Asaka T, Oda S, Kashiwada S, Mitani H (2018) $\gamma$-H2AX foci as indication for the DNA damage in 
erythrocytes of medaka (Oryzias latipes) intoxicated with 4nonylphenol. Environ Sci Pollut Res, 1-6

Sayour H, Kassem S, Canfarotta F, Czulak J, Mohamed M, Piletsky S (2019) Biocompatibility and biodistribution of surface-modified yttrium oxide nanoparticles for potential theranostic applications. Environ Sci Pollut Res, 1-13

Sharma N, Sharma M, Jamal QMS, Kamal MA, Akhtar S (2019) Nanoinformatics and biomolecular nanomodeling: a novel move en route for effective cancer treatment. Environ Sci Pollut Res:1-15

Zahin N, Anwar R, Tewari D, Kabir MT, Sajid A, Mathew B, Uddin MS, Aleya L, Abdel-Daim MM (2019) Nanoparticles and its biomedical applications in health and diseases: special focus on drug delivery. Environ Sci Pollut Res:1-18

Publisher's note Springer Nature remains neutral with regard to jurisdictional claims in published maps and institutional affiliations.

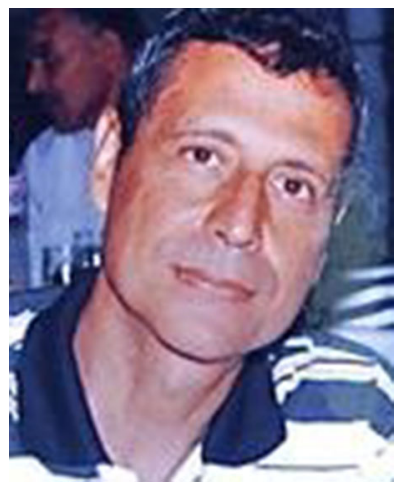

Dr. Lotfi Aleya is an Exceptional Class Professor of Biology, teaching at both the Faculty of Science and Faculty of Medicine, Besançon, France. He obtained his $\mathrm{Ph}$. D. from Blaise-Pascal University (National Center for Scientific Research, France). He published over than 300 papers on experimental studies and review articles at many peerreviewed journals related to microbiology, protistology, medicine, environmental neurology, harmful microorganisms, and ecotoxicology. He is Associate Editor of Science of the Total Environment, Editor of Environmental Science and Pollution Research, Review Editor of Inflammation Pharmacology, member of the Editorial Board Harmful Algae, of Sustainability, and Guest Editor of Oxidative Medicine and Cellular Longevity. He pilots cooperative projects in France and other countries, engaging private sector companies and public sector research centers associated with his Chair. He published many books and chapters and supervised $33 \mathrm{Ph}$. D thesis. Keywords for Research Interest Microbiology, Protistology, Medicine, Embryology, oxidative stress, Harmful microorganisms, Ecotoxicology.

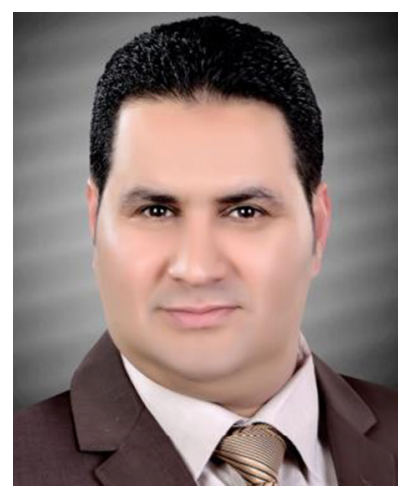

Dr. Mohamed Abdel-Daim is an associate professor of Pharmacology, Faculty of Veterinary Medicine, Suez Canal University, Ismailia, Egypt. Additionally, he serves as an associate professor at the Department of Zoology, Science College, King Saud University, Saudi Arabia. He is associate editor of Environmental Sciences and Pollution Research. He obtained his $\mathrm{Ph}$. D. from Kobe University Graduate School of Medicine, Japan. He published over than 170 papers on experimental studies and review articles at many ISI scientific journals related to ecotoxicology, pharmacology, drug safety, neuroscience, biochemistry, clinical pathology, molecular biology, and alternative medicine. He is a member of the Research Ethics Council, Academy of Scientific Research \& Technology, Egypt. Owing to the recognition of his research works, he has been awarded various research prizes, including the State Encouragement Award from the Academy of Scientific Research \& Technology, Cairo, Egypt, in 2014 and the First Class Medal of Excellence from the Presidency of the Arab Republic of Egypt on the national science day in August 2017. He served as an associate editor and had been a guest editor for 8 peer-review journal special issues. He also served as a reviewer for over 200 journals, as well as numerous national research grants. He has supervised over 30 Master and $\mathrm{PhD}$ theses. Keywords for Research Interest Environmental Pharmacology, Ecotoxicology, Alternative Medicine, Drug Toxicology, Neuropharmacology, Alternative Medicine. 